\section{International Scientific Journal Theoretical \& Applied Science}

p-ISSN: 2308-4944 (print) e-ISSN: 2409-0085 (online)

Year: $2014 \quad$ Issue: $10 \quad$ Volume: 18

Published: $30.10 .2014 \quad$ http://www.T-Science.org
Sergey Alexandrovich Mishchik Associate Professor,

Candidate of Pedagogical Science, Assistant professor Department of Physics, State Maritime University Admiral Ushakov, Russia, sergei_mishik@mail.ru

SECTION 21. Pedagogy. Psychology. Innovation in Education.

\title{
MATHEMATICAL MODELING HOLISTIC-SYSTEMIC COMMUNICATIVE ACTIVITY - THE THIRD TASK OF PEDAGOGOMETRIKS
}

Abstract: Proposed modeling holistic-systemic methods of mathematical communication activities regarding the integrity of the system analysis, system identity formation based on mathematical modeling of psychological and pedagogical theory of activity, psychological and pedagogical theory and system analysis stage formation of mental actions.

Key words: pedagogometrika, consistency, integrity, communication activities, game theory, analysis, forecasting, quality.

Language: Russian

Citation: Mishchik SA (2014) MATHEMATICAL MODELING HOLISTIC-SYSTEMIC COMMUNICATIVE ACTIVITY - THE THIRD TASK OF PEDAGOGOMETRIKS. ISJ Theoretical \& Applied Science 10 (18): 45-47. doi: http://dx.doi.org/10.15863/TAS.2014.10.18.11

УДК 372.851

\section{МАТЕМАТИЧЕСКОЕ МОДЕЛИРОВАНИЕ ЦЕЛОСТНО-СИСТЕМНОЙ КОММУНИКАТИВНОЙ ДЕЯТЕЛЬНОСТИ - ТРЕТЬЯ ЗАДАЧА ПЕДАГОГОМЕТРИКИ}

Аннотация: Предложено моделирование целостно-системной коммуникативной деятельности методами математического системного анализа относительно иелостно-системного формирования личности на основе математического моделирования психолого-педагогической теории деятельности, психолого-педагогического системного анализа и теории поэтапного формирования умственных действий.

Ключевые слова: педагогометрика, системность, целостность, коммуникативная деятельность, теория игр, анализ, прогноз, качество.

Проблема математического моделирования целостно-системной коммуникативной деятельности отражает стратегию совместного развития педагогометрических методов анализа целостно-системной жизнедеятельности, как циклического процесса, психологопедагогического системного анализа, теории формирования интеллекта и методов исследования операций с возможностью интерпретации выделенных процессов категориями теории игр. Реализация данных условий определяет третью задачу педагогометрики [1,2,3].

В общем случае математическая модель целостно-системной коммуникативной

\begin{abstract}
деятельности (ЦСКД) представляет многоуровневый образ, соответствующий различным социальным уровням - от личностных до международных отношений, при которых происходит обмен двенадцатью $(\mathrm{n}=12)$ предметно-деятельностными отношениями. В зависимости от социальной ситуации субъекты коммуникативной деятельности, зная на различном уровне структуру целостно-системного цикла жизнедеятельности (ЦСЦЖ), применяют свои возможности относительно позиционных игр, их стратегии, нормальной формы игры и контролем процесса соответствия. При этом позиционная игра $\mathrm{n}$ лиц устанавливает топологическое дерево $\Gamma$ c установленной
\end{abstract}


вершиной A, начальной структуры игры, функцией выигрыша, которая устанавливает каждой финишной позиции дерева $\Gamma$ n-вектор, разделение структуры всех компаундных позиций дерева $\Gamma$ на $\mathrm{n}+1$ множеств $\mathrm{S}_{0}, \mathrm{~S}_{1}, \ldots, \mathrm{S}_{\mathrm{n}}$, множества последовательности [4,5.6].

Стратегия игрока $i$, который воспроизводит структуру ЦСЦХ, есть функция, которая устанавливает перенос каждому информационному множеству $S_{i}^{j}$ этого игрока некоторый индекс из $I_{i}^{j}$. Множество всех стратегий игрока $i$ есть сумма величин $\sum i$. Если результаты случайных действий известны в вероятностном отношении, то представляем функции выигрыша как математическое ожидание при условии, что игрок $i$ применяет стратегию $\sigma \in$ $\sum i$ и применяем обозначение:

$$
\begin{aligned}
& \pi\left(\sigma_{1}, \sigma_{2}, \ldots, \sigma_{n}\right)= \\
& =\left(\pi_{1}\left(\sigma_{1}, \ldots, \sigma_{n}\right), \pi_{2}(\ldots), \ldots, \pi_{n}\left(\sigma_{1}, \ldots, \sigma_{n}\right)\right) \\
& \text { Функцию } \pi_{n}\left(\sigma_{1}, \ldots, \sigma_{n}\right) \text { на множестве всех } \\
& \text { возможных } \quad \text { значений }
\end{aligned}
$$
$\sigma_{1}, \sigma_{2}, \ldots \sigma_{n}$ можно выразить в форме соотношения или в виде n-мерной таблицы n-векторов. Тогда формируем n-мерную таблицу нормальной формой игры $Г$.

Любая целостно-системная коммуникативная деятельность, как игра $Г$, разложима в некоторой позиции $\mathrm{X}$ относительно ориентировочного, исполнительного и контрольного компонентов , если не существует информационных множеств, которые содержали бы позиции из двух множеств одновременно: 1) X и все следующие за ней позиции; 2) остальные позиции дерева игры. В этом случае надо выделить подигру $\Gamma_{x}$, состоящую из $\mathrm{X}$ всех следующих за ней позиций, и факторигру $\Gamma / \mathrm{X}$, состоящую из всех оставшихся позиций плюс $\mathrm{X}$, и функция выигрыша имеет вид $[7,8,9]$ :

$$
\pi_{X}\left(\sigma_{1 \mid \Gamma_{X}}, \sigma_{2 \mid \Gamma_{X}}, \ldots, \sigma_{n \mid \Gamma_{X}}\right) .
$$

Целостно-системная коммуникативная деятельность может принимать форму антагонистической игры, если существует $\left(p_{1}, \ldots, p_{n}\right)$ нулевая сумма удовлетворяет условию $\sum_{i=1}^{n} p_{i}=0$. Тогда $n$-компонента вектора выигрышей определяется остальными $n-1$ компонентами. В целом, нормальная форма конечной антагонистической игры приводится к матрице А с числом строк, равным числу действий игрока I, и с числом столбцов, равным числу действий игрока II.

При построении ориентировочного компонента ЦСКД возникают смешанные стратегии игрока как вероятностное распределение на множестве его чистых стратегий всех составляющих цикла. В этом случае, когда игрок имеет только конечное число $\mathrm{m}$ чистых стратегий, смешанная стратегия представляет собой $\mathrm{m}$-вектор $\mathrm{x}=\left(\mathrm{x}_{1}, \ldots, \mathrm{x}_{\mathrm{m}}\right)$,удовлетворяющий условиям $x \geq 0$ и $\sum_{i=1}^{m} x_{i}=1$. Если обозначить множество всех смешанных стратегий игрока I через X, а множество всех смешанных стратегий игрока II через У, и предположить, что игроки I и II участвуют в матричной игре A, то если игрок I выбирает смешанную стратегию X, а игрок II выбирает У, то ожидаемый выигрыш будет равен

$$
A(x, y)=\sum_{i=1}^{m} \sum_{j=1}^{n} x_{i} a_{i j} y_{j}
$$

или в матричной форме: $A(x, y)=x A y^{T}$.

Моделирование исполнительного компонента ЦСКД связывается с разработкой стратегией поведения, которые устанавливают набор $\mathrm{N}$ вероятностных распределений и задают возможные альтернативы в каждой информационной коммуникации. При этом возникает множество распределений А, что ни одно распределение из А не предпочитается обоими субъектами ЦСКД другому распределению из А, но для любого не входящего в А распределения $((x, y) ;(a-x, b-y))$ в множестве А найдется распределение $\left(\left(x^{\prime}, y^{\prime}\right) ;\left(\mathrm{a}-x^{\prime}, \mathrm{b}-y^{\prime}\right)\right)$, которое устанавливают оба субъекта ЦСКД.

Моделирование контрольного компонента ЦСКД определяется вектором значений игры, который задаёт $\mathrm{n}$-вектор $\varphi[\vartheta]$, удовлетворяющий аксиомам Шепли, выделяющих базисные условия математического моделирования целостносистемной коммуникативной деятельности педагогометрического анализа. Степень устойчивости ЦСКД задаёт норму поведения в форме коалиционной структуры в игре n-лиц. При этом возникает разбиение $\mathscr{T}=\left(T_{1}, T_{2}, \ldots, T_{m}\right)$ множества N. Данная структура представляет разбиение множества $\mathrm{N}$ на взаимно непересекающиеся коалиции. $\mathscr{T}$ Возникающая конфигурацией задаётся парой

$$
(x ; \mathscr{T})=\left(x_{1}, \ldots, x_{n} ; T_{1}, T_{2}, \ldots, T_{m}\right),
$$

где $\mathscr{T}$ - коалиционная структура, а $x$ представляет собой n-вектор, удовлетворяющий условиям

$$
\sum_{i \in T_{k}} x_{i}=v\left(T_{k}\right)
$$

для $k=1, \ldots, m$. Реализация выделенных условий приводит к установлению индивидуальной рациональности, выражающей системный тип ориентировки в целостно-системной коммуникативной деятельности и её математической модели. 


\section{References:}

1. Mishchik SA (2011) Proektirovanie matematicheskikh modeley fizicheskikh ob"ektov $\mathrm{v}$ protsesse formirovaniya tselostno-sistemnoy samostoyatel'noy uchebnoy deyatel'nosti. Odinnadtsataya mezhdunarodnaya konferentsiya "Fizika v sisteme sovremennogo obrazovaniya", FSSO-11, 1 tom - Volgograd: Izd-vo VGPU, 318.

2. Mishchik SA (2012) Organizatsiya laboratornogo fizicheskogo praktikuma na baze mobil'nykh programm platformy android $\mathrm{V}$ protsesse tselostno-sistemnoy shirokoprofil'noy podgotovki. XII Mezhdunarodnaya uchebno-metodicheskaya konferentsiya "Sovremennyy fizicheskiy praktikum”, Moscow, 25-27 sentyabrya 2012 goda. - Moscow: - Izd-vo MGTU im. N.E. Baumana, 325.

3. Mishchik SA (2014) Tselostno-sistemnyy tsikl uchebnoy zhiznedeyatel'nosti - model' professional'noy deyatel'nosti shirokoprofil'nogo spetsialista. Materialy Mezhdunarodnoy nauchnoy konferentsii «Deyatel'nostnaya teoriya ucheniya: sovremennoe sostoyanie i perspektivy», Moscow. 6-8 feb 2014. Moscow: Izdatel'stvo Moskovskogo universiteta, 384.

4. Mishchik SA (2014) Bazisnost'. Fundamental'nost'.

Pedagogometrichnost. Shirokoprofil'nost'. Materialy Mezhdunarodnoy nauchnoy konferentsii «Moderni vymozenosti vedy - 2014». - Dil 16. Pedagogika.: Praha. Publishing House «Education and Science» s.r.o, 112.

5. Mishchik SA (2014) Modelirovanie shirokoprofil'noy tselostno-sistemnoy deyatel'nosti. Materialy II Mezhdunarodnoy nauchnoy konferentsii «Prioritety mirovoy nauki: eksperiment i nauchnaya diskussiya»: 24-25 dec 2013. S -Peterburg North Charleston, SC, USA: CreateSpace, 151.

6. Mishchik SA (2013) Formirovanie tselostnosistemnogo tsikla uchebnoy zhiznedeyatel'nosti shirokoprofil'nogo spetsialista metodami matematicheskogo modelirovaniya. Sbornik materialov 3-y mezhdunarodnoy nauchnoprakticheskoy konferentsii. 2 chast'. Problemy sovremennoy nauki v 21 veke. Makhachkala, 28 dec 2013: - Makhachkala: OOO «Aprobatsiya», 195.

7. Mishchik SA (2014) Strukturnoe formirovanie pedagogometricheskikh funktsiy matematicheskogo analiza tselostno-sistemnogo uchebnogo protsessa. Materialy Mezhdunarodnoy nauchnoy konferentsii «Nastoyashchi izsledvaniya i razvitie - 2014» 17-25 jan 2014. Tom 14. Pedagogicheski nauki. - Sofiya, 2014: «Byal GRAD-BG» OOD, 96.

8. Mishchik SA (2014) Pedagogometrika i matematicheskoe modelirovanie uchebnoy deyatel'nosti. Materialy Mezhdunarodnoy nauchnoy konferentsii «Modern mathematics in science» - 30.06.2014. ISJ Theoretical \& Applied Science 6(14): 54-56. - Caracas, Venezuela. doi: http://dx.doi.org/10.15863/TAS.2014.06.14.10

9. Tokmazov GV (2014) Matematicheskoe modelirovanie v uchebno-professional'noy deyatel'nosti. Materialy Mezhdunarodnoy nauchnoy konferentsii «Modern mathematics in science» - 30.06.2014. ISJ Theoretical \& Applied Science 6(14): 44-46. - Caracas, Venezuela. doi: http://dx.doi.org/10.15863/TAS.2014.06.14.8 